\title{
LA CIUDAD, LOS JÓVENES Y EL CAMPO DE LAS PRÁCTICAS CORPORALES
}

\author{
MS. GABRIEL ARMANDO CACHORRO
}

Magíster en desarrollo educativo - Universidad Pedagógica Nacional de México

Profesor Adjunto de la cátedra Educación física I y seminario tesis - Universidad Nacional de La Plata

(Buenos Aires - Argentina)

Email: gcachorro@yahoo.com

\section{MS. ALDO ROMÁN CÉSARO}

Magíster en educación corporal - Universidad Nacional de La Plata

Profesor de juego y recreación en la cátedra Educación física 1 y 2

Profesor de teoría de la educación física 4 - Universidad Nacional de La Plata

(Buenos Aires - Argentina)

E-mail: rancull@hotmail.com

\section{GRAD. MARTÍN SCARNATTO}

Maestrando en educación corporal - Universidad Nacional de La Plata

Profesor de didáctica especial I (Fahce)

Profesor de historia social del deporte - Facultad de Periodismo y comunicación social - Universidad

Nacional de La Plata (Buenos Aires - Argentina)

E-mail: scarnatto@gmail.com

\section{GRAD. JUAN PABLO VILLAGRÁN}

Profesor del seminario tesis en el profesorado y la licenciatura en educación física - Universidad

Nacional de La Plata (Buenos Aires - Argentina)

E-mail: teclajuan@yahoo.com.ar

\section{RESUMEN}

La investigación abordó una reconstrucción del campo de las prácticas corporales en la ciudad de la Plata.

Los referentes empíricos, sujetos juveniles, protagonistas de la resignificación de los espacios de la ciudad para el despliegue de prácticas corporales, del trazado de diversos territorios de interacción y rituales de la comunicación corporal.

La estrategia involucra: a. Rastrillaje de prácticas, territorios y saberes corporales existentes en la ciudad, mediante censos y encuestas; b. aplicación de registros etnográficos; c. triangulación de los datos obtenidos; d. clasificación e inventariado de las propuestas corporales.

PALABRAS CLAVES: Ciudad; juventud; prácticas corporales; campo. 


\section{INTRODUCCIÓN}

El siguiente artículo ofrece algunas problematizaciones acerca del estado actual del campo de las prácticas corporales de los jóvenes en la ciudad de la Plata. Se pretende actualizar estos datos ante la emergencia de nuevas tendencias de la cultura corporal, que sedimentan otros patrimonios corporales en los sujetos que habitan las ciudades. En los rastrillajes previos puede constatarse que, los abordajes de este tema, no han sido desarrollados desde disciplinas como la educación física.

Solo puede identificarse en la profesión citada, un inventario de las prácticas corporales y un criterio de clasificación que delinea enfoques psicomotrices, físico deportivos y pedagógicos.

En este criterio de agrupación no están empadronadas un conjunto de prácticas y saberes corporales más recientes (danzas árabes, murgas, pilates) o aquellas configuraciones particulares del movimiento, engendradas en la mezcla de disciplinas corporales procedentes de orígenes geográficos distintos (tae bo, capoeira, taekwondo etc.).

Las transformaciones de los capitales corporales existentes en la cultura, se expresan en las vocaciones corporales que incuban los sujetos juveniles en sus procesos de interacción con las realidades donde participan. La diversificación de estas opciones de adscripción a ofertas del cuerpo en movimiento, nos muestra una profundización de la distancia entre, las propuestas de educación corporal tradicionales (deporte, gimnasia, juego, natación) de instituciones formadoras y las nuevas preferencias corporales de las generaciones juveniles entre las que podríamos agregar, malabarismos, acrobacias, bailes centroamericanos $y$ otras actividades alternativas.

\section{MARCANDO EL ÁREA}

Se desprende de una necesidad de entender y explicar las transformaciones en campo de las prácticas y saberes corporales, producidas en la vida de la ciudad. La aceleración de estos procesos de cambio expresados en la cultura corporal, carece de estudios o de trabajos de indagación y sistematización para la construcción de marcos explicativos de estos temas emergentes. Entendemos que el campo de las prácticas corporales que devienen en una ciudad, poseen una composición abierta a sucesivas modificaciones propias de la cultura.

La capacidad instituyente de sus componentes, cuerpo y cultura, muestran a los contenidos de la educación física, rezagada respecto de estas revoluciones del campo y sus prácticas corporales emergentes. La investigación del estado actual de 
las prácticas corporales en el campo, es una dimensión postergada que merecen ser atendidos. La inscripción en esta área de interés, se fundamenta en el corto alcance descriptivo y explicativo de los abordajes físico-deportivo, psicomotriz o pedagógico del cuerpo en la educación física (CRISORIO, I995; VázQUez GómeZ, 1989; y otros), para atender las recomposiciones de las propuestas corporales del campo. Sus alcances teóricos se tornan insuficientes para dar cuenta de la trama compleja que se configura en la copresencia de un vasto catalogo de propuestas de actividades corporales que pugnan por obtener sitios de aprobación y reconocimiento social.

El campo de las prácticas y saberes corporales, aparecen como un objeto de estudio, poco explorados desde la disciplina educación física. Son sitios donde se pueden construir y sistematizar la reformulación de nuevas prácticas y saberes producidas por la capacidad generativa incesante, del cuerpo y la cultura en la ciudad.

\section{INDAGACIONES PRELIMINARES}

El campo de las prácticas corporales, ha sido interpretado por los diseñadores del curriculum de la educación física a través de un criterio de selección que jerarquiza un conjunto de configuraciones del movimiento de relevancia y significatividad social en la sociedad. Los contenidos escogidos por los productores del conocimiento corporal, son: deporte, gimnasia, juego, natación y vida en la naturaleza. La arbitrariedad cultural de esta selección, no invalida la existencia de otra variada gama de alternativas corporales, surgidas de la capacidad instituyente de la cultura.

A partir de aquí podemos referirnos al campo de las prácticas y saberes corporales, más allá de los lineamientos curriculares vigentes. El campo de la cultura corporal, se manifiesta en una perpetua recomposición de la ubicación de una variada gama de actividades corporales (cuyo amplio espectro va del patín a las artes marciales). Las prácticas corporales se producen en un intercambio y mutua afección, muchas veces conflictiva, con otras prácticas. El resultado de esta relación es una hibridación de capitales corporales, que proceden de distintos deportes, gimnasias, danzas.

Estas prácticas sufren mutaciones que no se dan en el vacío, sino que tienen anclaje en lugares de realización muy concretos - una plaza, un ciber, una discoteca, un club como lugares de posibles donde se condensan estos procesos sociales. Asimismo los jóvenes ocupan territorios, hacen puestas en escena en un momento histórico complejo - de mundialización de las culturas. La reflexividad de estas prácticas corporales, los desplazamientos y recomposiciones, están producidas por los sujetos, y en especial los sujetos juveniles, quienes participan en los diversos 
casilleros de la vida social, en tanto son las generaciones que aportan nuevos sentidos y significados al amplio espectro de propuestas emergentes.

En este proceso de cambio se produce una reformulación del campo, que se materializa en la inauguración de un nuevo conjunto de capitales corporales, donde se incluye el repertorio de motricidades, gestos y movimientos. Este proceso de transformación, demanda una investigación que detecte las particularidades y detalles de estas manifestaciones corporales, con una mirada alternativa del cuerpo y la cultura, haciendo un anclaje en las actuaciones de los jóvenes, sus puestas en escena, la localización de sus territorios de actuación, sus disputas con la parcela de la realidad donde se encuentran situados.

Hasta el momento no se detectaron trabajos de investigación previos sobre la especificidad de esta temática. En el proceso de recolección de información para armar el "estado de la cuestión" se pueden registrar trabajos relacionados con nuestro objeto de estudio. El campo de las prácticas corporales, si bien constituye un problema de relevancia social, no aparece como tema de investigación preciso y delimitado.

La emergencia de prácticas y saberes de la cultura corporal se expresa en los diversos sectores del campo, sin haber sido sometidos a tratamientos y estudios rigurosos, capaces de clasificar, sistematizar y ordenar los datos procedentes de las distintas realidades corporales.

Las transformaciones de la cultura corporal, expresadas en las luchas por capitales corporales y las tensiones por el reconocimiento simbólico, tampoco son estudiadas y no se hallan descripciones acerca de las lógicas de operación con las que se manifiestan sus fuerzas configuradoras del espacio social del cuerpo. La producción de materiales bibliográficos existentes, no son específicos y aparecen incompletos.

La ausencia de estudios particulares sobre este tópico, habilita la recolección de materiales procedentes de distintos paraderos disciplinares, para generar una amalgama de ideas capaces de interpretar los procesos configuradores de estos saberes corporales. Las distintas realidades pueden ser analizadas, desde una perspectiva multidisciplinaria que integre las categorías sociológicas de Bourdieu sobre campo, habitus, prácticas, trayectorias, capitales. La aplicación de este marco teórico posibilita una reconstrucción del campo específico de las prácticas corporales, identificando sus estructuras organizadoras.

La identificación de los elementos que lo componen puede ser completada, con el estudio de las lógicas internas que operan en su interior, dando entrada a diversas perspectivas teóricas de la antropología del cuerpo aportadas por Le Bretón (1990), los ensayos de producción de cuerpos en el contexto de la modernidad, 
vertidos por Lipovetsky (1988), Giddens (1999), Heller y Fehér (1995), Maffesoli (2004).

Las diferentes miradas sobre el cuerpo y la cultura se cristalizan en grupos sociales localizados en condiciones específicas de realización social. Los sujetos juveniles son referentes empíricos que inauguran nuevos modos corporales de asumir sus posiciones en el mundo.

Estas tendencias del cuerpo y la cultura se aprecia, en la formación de tribus urbanas (Margulis, 2003; Sarlo, 1996; Reguillo Cruz, 2000) las "socialidades múltiples" de los sujetos posmodernos desplegando celebraciones corporales en fiestas rave, bailes callejeros (MAFFESOLI, 200 I), la construcción social de un "nuevo sensorium" de las sensaciones corporales, mediados por las nuevas tecnologías de la comunicación (MARTín BARBERO, 1998).

Estos trabajos poseen elementos explicativos, de las tendencias de los sujetos a involucrarse en el despliegue de prácticas comunes y además la emergencia de rituales corporales, nos aportan datos empíricos valiosos para entender los sentidos y significados que los actores les atribuyen a estos modos de participar a través del cuerpo.

\section{PERSPECTIVAS Y MATERIALES TEÓRICOS}

El marco teórico de este artículo presenta los temas: cuerpo y cultura. La cultura corporal se distancia de la "cultura física" (CAGIGAL, 1983) por el estrecho alcance de esta construcción lingüística que solo se circunscribe a un número mensurable de actividades corporales y motrices tradicionales (atletismo, fútbol, básquetbol etc.), poniendo el acento en dimensiones biomecánicas del movimiento útiles para ejecutar los sistemas técnicos y tácticos del juego.

Nuestra mirada se inscribe en la perspectiva de los estudios culturales localizados en la diversidad de problemas emergentes del cuerpo en la trama de la cultura.

Las transformaciones del campo de las prácticas corporales tienen un sitio privilegiado de materialización, los terrenos conflictivos de la educación física y los territorios de la ciudad. Los análisis sobre los capitales corporales viejos y nuevos puestos en relaciones de lucha por su legitimidad, tienen a los sujetos juveniles como referente empíricos que condensan los profundos cambios en la cultura corporal, que pueden identificarse claramente en las tendencias de las modas, las preferencias sexuales, los hábitos corporales, los gustos deportivos, las estéticas corporales, la educación de imágenes corporales tecnológicas, nuevos comportamientos corporales. Todas estas manifestaciones de revolución de los cuerpos colectivos, puede localizarse en las murgas, el hip hop, el skate, el turismo aventura. 
En este sentido, colocando como telón de fondo la cultura corporal, el proyecto aborda las transformaciones en el campo, de las prácticas y saberes corporales. Estos cambios se estudian tomando como referencia a los sujetos juveniles y haciendo anclaje en la comunicación, las prácticas y los territorios que se construyen el la ciudad.

La categoría teórica de cuerpo es entendida como el modelaje particular que hace la cultura sobre la materialidad física de los sujetos. El cuerpo no es el soma sino las posibles configuraciones sociales que puede adoptar esa materia viva. El cuerpo desde esta perspectiva es una construcción social anclada en un tiempo y espacio específico, resulta de esa interacción dialéctica con otros cuerpos y con la realidad. Por el nivel de complejidad que contempla el cuerpo, efectuamos un tratamiento con el aporte de miradas complementarias entre Bourdieu (1990), De Certeau (1996) y Le Breton (1999).

El concepto de campo es tomado de Pierre Bourdieu ( 1990) como un recorte analítico del espacio social, donde los actores de las prácticas y saberes corporales libran luchas materiales y simbólicas por la apropiación de capitales corporales. El devenir del campo muestra la participación de agentes que adoptan posiciones, despliegan trayectorias, ponen en juego sus tácticas y estrategias de conservación o de innovación a partir de una "illusio" (convicción que vale la pena, hacerse cargo de esa disputa por el reconocimiento y legitimación dentro de ese recinto específico de las prácticas y saberes del cuerpo).

La noción ciudad es entendida como un soporte material indispensable donde se expresan las prácticas corporales adoptando configuraciones diversas, condicionadas muchas veces por las características del paisaje. Es un mapa con dimensiones geográficas objetivas, con sedes, paradas, regiones, zonas. La posesión de estos elementos estructurales de la ciudad, van acompañados de propiedades dinámicas que le imprimen los actores en el trazado de trayectorias cotidianas, los desplazamientos por sus espacios urbanos, las migraciones de un sitio a otro, la ocupación de territorios. La ciudad, es posible de ser cartografiadas por las apropiaciones subjetivas de los sujetos ciudadanos que la habitan con criterios de usos y valoraciones heterogéneos. Esta experiencia ciudadana brinda una construcción del cuerpo y la cultura de sus moradores. Existen estudios sobre este tópico (GARCía CANCLINI, 2004; AugE, 2000), que muestran desde enfoques diversos, este fenómeno de la constitución de los sujetos en la ciudad.

En este texto se utiliza la noción de "jóvenes" porque ella se desprende de estudios de corte cualitativo describiendo los procesos culturales evitando la generalización de estos sujetos teniendo en cuenta sus múltiples modos de vivir sus cuerpos atravesados por situaciones particulares de existencia (FEIXA, 2006). La noción de 
cultura juvenil rescatada por Rosana Reguillo Cruz (2000), nos permite establecer una clara diferenciación de la noción "adolescente" que en cambio propone una descripción estandarizada del desarrollo evolutivo de los aparatos - reproductor masculino y femenino - y sistemas - nervioso, cardiorespiratorio - del ser humano dotado de un físico. Las diversos desacoples de esos sistemas desencadenan la constitución de la identidad personal en medio de crisis.

Los cuerpos juveniles nos ofrecen diversas dimensiones posibles de ser abordadas; en este trabajo se pretende analizar los procesos de cambio corporal en los modos de comunicación, las formas de ocupar los escenarios de interacción social y las maneras de desplegar prácticas corporales.

En lo referente a la comunicación la entendemos como una producción social de sentidos y significados negociados por los sujetos. En esta orquestación de las prácticas de la comunicación, prestamos especial atención a los enfoques de Erving Goffman (1989) y de Edward Hall (1987) que se instalan en la comunicación interpersonal de los sujetos resaltando su dimensión corporal en las actuaciones. Allí, analizamos la puesta en juego del lenguaje de los signos corporales, el desarrollo de la metacomunicación (BATESON, 1985) puestas en claves de comunicación no verbales. Es oportuno señalar que, establecemos un distanciamiento de la comunicación y contracomunicación motriz aprovisionada por la psicomotricidad o la sociomotricidad, (Le BOULCH, I99I; PARLEBAS, 1993) en tanto esta vertiente de la educación física, solo se enmarca en recintos cerrados de las competencias deportivas resaltando el interés por dimensiones instrumentales de los dispositivos técnicos y la tácticas de los competidores.

Con respecto a los territorios, nos da la posibilidad de abordar el espacio rompiendo su dureza. El territorio es una palabra que refiere a la puesta en escena, la actuación de los actores en un espacio que se hace móvil, cambiante y se muestra en permanente reconfiguración según las circunstancias azarosas y contingentes que el devenir depara. Es un territorio complejo que improvisa situaciones tradicionales cara a cara y las entrecruza con escenarios virtuales o también con escenarios de los encuentros multiculturales. Acerca de este concepto recurrimos a los aportes de Renato Ortiz (1997) y Michelle De Certeau (1996).

Con respecto a las "prácticas corporales" las entendemos como "configuraciones particulares de movimiento", según la cultura donde se exprese. Esa idea se fundamenta en la propuesta de Norbert Elias y Eric Dunning (1996) quien habla acerca de la "figuración", la relevancia social de un juego, la danza, el deporte, modelado por una cultura particular, y condicionado por sus expectativas sociales regionales. Las prácticas corporales, adquieren sentido, proyección y significado 
para los sujetos porque están dirigidas por una matriz de percepción, pensamiento y acción lo que Bourdieu ( 1990) define como un "habitus" con su poder organizador que opera como un factor disposicional o una estructura estructurante que para nuestro interés indagatorio, se concreta en el cuerpo.

En lo que concierne a los "saberes corporales" son producidos en el protagonismo de los cuerpos. Son resultados inacabados, están en perpetua reformulación, se derivan de la participación de los sujetos con sus cuerpos en distintas realidades. El patrimonio de la cultura corporal surge de la relación y elaboración de los sujetos con la cultura, en el despliegue de prácticas corporales en el contexto de la modernidad. En esa situación de elaboración reflexiva del cuerpo, trazado en planos deportivos, religiosos, sexuales, se sedimentan saberes corporales en un acervo de experiencias sociales (ScHüTz, 1974) que varían su riqueza y nivel de especialización, de acuerdo a los niveles de intromisión de los sujetos en la cultura, y las incursiones en ofertas corporales a la carta. Las elecciones están a la medida de las expectativas sociales de los sujetos (spa, reiki, aladelta, yoga, spinning, boxeo, tenis, winserf, parkour, hip-hop etc.).

\section{APORTE ORIGINAL AL TEMA}

El empadronamiento de las nuevas prácticas y saberes corporales, emergentes de los procesos de cambio inherentes a la cultura corporal puede significar un insumo valioso, para actualizar los contenidos tradicionales de la educación física jerarquizados para su transmisión.

Un estudio que trace un diagnostico, relevamiento de las prácticas y saberes corporales participantes del campo de la cultura corporal, se impone ante la crisis de sentido que expresa las propuestas de educación física con las vocaciones corporales de las nuevas generaciones.

El cuerpo y la cultura son los cimientos sobre donde posan las clases de educación física, puestas en escena entre los participantes de la interacción social. El abordaje de la educación física como práctica social donde se materializan las nociones de cuerpo y cultura. Sitio donde podemos ver como se expresan las nociones de cuerpo y subjetividad mostrándonos sus propiedades, relaciones y las tensiones de estos conceptos.

Cuerpo y cultura, son categorías teóricas de elevado nivel de abstracción pero que pueden someterse a rigurosos análisis empíricos si tomamos como lugares de anclaje concretos, las prácticas corporales que despliegan una muestra acotada de sujetos juveniles. Presentando un seguimiento en zonas de la ciudad donde se engendran practicas corporales significativas y relevantes en la actualidad. La selección 
de parcelas de la realidad donde se desenvuelven los jóvenes, podremos definir, caracterizar, identificar, retratar estos cambios del cuerpo y la cultura, en coordenadas de espacio y tiempo especificadas en regiones particulares de la ciudad.

\section{BÚSQUEDAS EN LA CIUDAD}

La ciudad puede ser asumida inaugurando otros senderos, frecuentando otros lugares o proponiendo otros ángulos de apreciación de sus paisajes. La búsqueda de otra perspectiva de contemplación de las prácticas y saberes corporales de los sujetos juveniles demanda una posición abierta y sensible para poder entender los procesos sociales y desde esta óptica de apreciación avanzar en a producción una sistematización de datos que describa el campo de las prácticas y saberes corporales existente en la ciudad de La Plata.

La explicitación de nuestra matriz cultural desde donde focalizamos los cuerpos en la ciudad nos ayuda a definir las categorías cuerpo y cultura, sus rasgos y propiedades que asumen en el campo disciplinar de la educación física. Describir las características particulares que adoptan la diversidad de prácticas corporales de la ciudad de La Plata, con una perspectiva de abordaje permeable al devenir de los cuerpos en la ciudad nos facilita el descubrir las relaciones y tensiones que se manifiestan entre el cuerpo y la cultura de los sujetos juveniles platenses.

Las búsquedas no son inocentes, exigen una confesión teórica y a partir de esta revisión de nuestros marcos conceptuales desde donde recortamos la realidad podemos identificar los procesos de permanencia y cambio del cuerpo y la cultura que expresan los sujetos juveniles en el despliegue de sus prácticas corporales. Construir categorías de observación teórica del cuerpo y la cultura para reinterpretar las prácticas tradicionales de la educación física.

\section{DESPLAZAMIENTOS EN LA CIUDAD}

Nuestro enfoque se inscribe en la perspectiva mixta de investigación social que apela a recursos cuantitativos y cualitativos, desarrollando un abordaje de escala macrosocial. Las razones de esta elección de corte cualitativo, se explica porque se pretende reconstruir el campo de las prácticas corporales existentes en la ciudad de La Plata e indagar las nociones del cuerpo y cultura, en la especificidad de las prácticas orquestadas por los habitantes de una ciudad, desde una mirada cultural que interpreta sentidos sociales acordados por sujetos juveniles, la construcción de códigos de comunicación corporal, la emergencia de nuevas preferencias corporales, las apropiaciones subjetivas de territorios. 
Por las intenciones de búsqueda de este trabajo, se apela a una intervención de corte antropológico y etnográfico. Los motivos por los que se utilizan recursos de la metodología cuantitativa, se debe a la necesidad de empadronar regiones, encontrar datos estadísticos de las propuestas corporales existentes en el mapa de la ciudad, haciendo una cartografía de las distintas regiones donde los sujetos se convocan para desplegar las prácticas corporales.

La perspectiva cuantitativa de investigación por su modalidad estructurada y sistemática en la forma de recolectar los datos, es adecuada para obtener un panorama macrosocial de la ciudad y de este registro seleccionar pequeñas parcelas de realidad con grupos etarios especificados en el tiempo y el espacio. En los sitios de realización social elegidos como muestras significativas, por su pertinencia para el objeto de estudio de investigación, se estudian los procesos de cambios en el devenir de las prácticas corporales. El seguimiento de las transformaciones del cuerpo y la cultura localizado en los sujetos juveniles, utilizando instrumentos estandarizados luego es profundizado con la inclusión de los procesos interactivos de los sujetos estudiados.

La triangulación entre métodos rescata el enfoque cualitativo como forma de abordaje porque esta alternativa, contiene técnicas de recolección de datos flexibles y adaptables a los diversos momentos de la investigación. Se apela al uso de diversas técnicas de recolección de información según los objetivos trazados en cada etapa de la investigación. El enfoque cuantitativo nos posibilita a través de encuestas y censos, inventariar los lugares de realización de las prácticas corporales en la ciudad, identificar los sujetos juveniles participantes, trazando un mapa con regiones diferenciadas.

\section{OPERACIONES EN CAMPO}

En una primera etapa se desarrollaron tareas exploratorias del objeto de estudio a indagar, para obtener una noción panorámica de la realidad donde se localizan los sujetos juveniles y sus preferencias corporales, en los distintos espacios de la ciudad. Es decir se hizo una primera aproximación aplicando cuestionarios para detectar tendencias o perfiles que orientan la construcción de vocaciones corporales.

Se planificaron recorridos por distintos sitios de la ciudad para identificar lugares donde puedan obtenerse información sobre sedes donde se desarrollan prácticas corporales relevantes para los objetivos e esta investigación.

La detección de sitios de reunión social para la puesta en escena de prácticas corporales (gimnasios, estéticas corporales, teatro corporal, danza contemporánea etc.) se tomaron como lugares para la aplicación de un censo, con la pretensión de 
obtener datos iniciales, rasgos generales de las propuestas de diversas organizaciones o empresas relacionadas con el despliegue de prácticas corporales.

Existen datos municipales sistematizados y empadronados sobre agencias dedicadas a distintos tratamientos y ofertas corporales (salones de belleza, centros de rehabilitación, gimnasios, escuelas de danzas, clubes de barrio). A la vez existen territorios no declarados, caracterizados por la informalidad de los encuentros donde también existen producciones de sentidos y significados en la organización de encuentros entre sujetos, donde se comparten prácticas corporales específicas (las plazas, las ramblas, los parques, esquinas peculiares, galpones).

La recolección de materiales oficiales es una puerta de acceso a la reconstrucción del campo y el rastreo de sitios no declarados en documentos escritos, es otra vía de entrada que demanda otra lógica de armado de los datos.

Las observaciones no participantes, nos sirven para trazar relevamientos de los diversos territorios sociales de actuación y dentro de esos espacios sociales trazar un primer esbozo, cartografiar los escenarios de participación y registrar las formas del lenguaje corporal empleados por los jóvenes. Las encuestas nos permiten graficar una visión general de las actividades corporales que están en apogeo en los sujetos juveniles.

En una segunda etapa se proyectó un acercamiento a mayor profundidad en el trabajo de campo. Se apeló a registros audiovisuales porque este instrumento nos otorga la posibilidad de capturar el movimiento del cuerpo, congelarlo, someterlo a visionados aplicando la "observación diferida", rescatando las formas de trabajo de los estudiosos de la comunicación no verbal pero modificando el tipo de observación. Este recurso entendemos pertinente por la lógica de la expresión corporal que se constituye en lo imprevisible, cambiante, veloz y nos demanda para su análisis minucioso y detallado una grabación de imágenes corporales de los jóvenes.

Se utilizan entrevistas a jóvenes para acceder a los sentidos sociales que ellos les atribuyen a sus prácticas corporales, las valoraciones de los territorios y los significados subjetivos que le otorgan a las prácticas y saberes corporales. Entendemos importante la representación personal que hacen los sujetos de sus propias actuaciones corporales que no pueden ser caracterizadas por sujetos que no ponen el propio cuerpo en esa experiencia juvenil. Entendemos como un requisito central la experiencia corporal para poder hablar desde el atravesamiento.

La puesta en acto de la observación participante nos permite acceder a las sensaciones kinestésicas y tener una mayor proximidad con la percepción de la realidad que los cuerpos juveniles crean en sus vidas cotidianas. La participación en celebraciones corporales colectivas, la entrada y vivencia de instancias de la cultura corporal ajena a la de los investigadores reporta un saber que trasciende la empatía. 
Estar en el bastidor de la murga, el circo, la comparsa, en la preparación de una marcha que implica un trabajo de composición del cuerpo colectivo es otra puerta de acceso a la comprensión de las prácticas y saberes corporales.

\section{REFLEXIONES EN CAMPO}

A modo de cierre elegimos esbozar las siguientes ideas en torno a la ciudad, los jóvenes y las prácticas corporales:

a. Existe un empadronamiento del campo de las prácticas corporales sin actualizar e incluir en el ámbito de la educación física. Las prácticas corporales urbanas ofrecen grupos de practicantes con un espectro diverso (parkour, malabares, circo, murga, equitación, buceo, aladelta) desligado de los planes de estudio de la educación física. Este proceso deja entrever una crisis de los contenidos tradicionales de la educación física, envejecidos ante la aparición de propuestas más atractivas para los deseos de los sujetos juveniles.

b. Se identifica una emergencia de nuevas prácticas y saberes corporales, dentro del campo del cuerpo y la cultura. Alcanzan autonomía y complejidad gestualidades, corporalidades y motricidades urbanas por espacios de participación ciudadana contiguas. Se manifiestan como vidas urbanas paralelas que no se integran. Por ejemplo en las formas de baile y performances callejeras. Los jóvenes están inaugurando nuevas tendencias corporales y tienen la posibilidad de acceso a múltiples ofertas corporales. Que implican un proceso de recomposición de las prácticas corporales tradicionales, materializada en los modos subjetivos que los jóvenes la asumen.

c. La ubicación del cuerpo en los escenarios de participación social se ven afectados por el proceso de mundialización de las culturas. Los sujetos juveniles reformulan sus acervos de experiencias corporales y sus matrices de formación corporal, ante el enfrentamiento con nuevas prácticas y saberes corporales en un contexto de modernidad mundo y desencadenan composiciones, combinaciones de las influencias dinámicas y cambiantes. Las prácticas y saberes corporales que se expresan en el campo de la cultura corporal se enmarcan en la biopolítica. Las prácticas corporales y motrices se intercalan con grafittis, tatuajes, ritmos musicales, oralidades 
callejeras, cadencias del movimiento, hábitos alimenticios, formas de sensualidad. Ellas condensan sentidos estéticos y políticos ante la vida puesta en los laberintos la ciudad.

\section{A cidade, os jovens e o campo das práticas corporais}

RESUMO: A investigação aborda uma reconstrução do campo das práticas corporais na cidade de La Plata.

Os referentes empíricos, sujetos juvenis, protagonistas da ressignificação dos espaços da cidade para o desdobramento de práticas corporais, do traçado de diversos territórios de interação e rituais da comunicação corporal.

A estratégia envolve: a. uma pesquisa das práticas, os territórios e saberes corporais existentes na cidade, mediante censos e enquetes; $b$. aplicação de registros etnográficos; c. triangulação dos dados obtidos; d. classificação e inventariado das propostas corporais.

PALAVRAS-CHAVE: Cidade; juventude; práticas corporais; campo.

\section{Bodily practices, the youths and the city}

ABSTRACT: The research approaches a reconstruction of the field of the bodily practices in the city of La Plata.

The empirical modals are juvenile subjects, since they have a leading role in establishing bodily practices, generating different body expression rituals and interaction areas.

The working strategy involves: a. a field study of the practices, areas and knowledge of bodily practices in the city, by means of censuses and surveys; b. using ethnographic records, c. triangulation of data obtained; d. classification and inventory of the different bodily options.

KEY WORDS: City; young subjects; bodily practices; field.

\section{REFERENCIAS}

ANZIEU, D. El yo piel. Madrid: Biblioteca Nueva, 1987.

AUGE, M. Los no lugares. Barcelona: Gedisa, 2000.

BATESON, G. Pasos hacia una ecología de la mente. Una aproximación recoluvionaria a la autocomprensión del hombre. Buenos Aires: Planeta Carlos Lohle, 1985.

BERNARD, M. El cuerpo. Buenos. Aires: Paidós, 1985.

BLEICHMAR, S. La subjetividad en riesgo. Buenos Aires: Topia Editorial, 2005.

BOURDIEU, P. La juventud es más que una palabra. In: Sociología y cultura. D.F:: Grijalbo CNCA, 1990.

BRACHT, V. Educación física y aprendizaje social. Córdoba: Vélez Sársfield, 1996. 
CAGIGAL, J. En torno a la educación por el movimiento. Revista Stadium, Buenos Aires, n. 96, p. 33-44, 1983.

CHOKLER, M. H. Los organizadores del desarrollo psicomotor. Del mecanismo a la psicomotricidad operativa. Buenos Aires: Ediciones Cinco, 1988.

CRISORIO, R. Enfoques para el abordaje de los cbc desde la Educación Física. Revista Serie Pedagógica, n², Facultad de Humanidades y Ciencias de la Educación, UNLP, Buenos Aires, p. 175-192, 1995.

DAVIS, F. La comunicación no verbal. Madrid: Alianza Editorial, 1991.

DE CERTEAU, M. La escritura de la historia. D.F:: UIA, 1996. - La invención de lo cotidiano. Artes de hacer. D.F.: U.I.A, 1998.

ESPOSITO, R. Immunitas. Protección y negación de la vida. Buenos Aires: Amorrortu Editores, 2005.

ELIAS, N.; DUNNING, E. Deporte y ocio en el proceso de la civilización. DF: Fondo de Cultura Económica, 1996.

FOUCAULT, M. Microfísica del poder. Madrid: La Piqueta, 1992.

FEIXA, C. De jóvenes, bandas y tribus. Antropología de la juventud. Barcelona: Ariel, 2006.

GARCÍA CANCLINI, N. Diferentes, desiguales y desconectados. Mapas de la interculturalidad. Buenos Aires: Gedisa, 2004.

GIDDENS, A. Modernidad e identidad del yo. Buenos Aires: Península, 1999.

GOFFMAN, E. La presentación de la persona en la vida cotidiana. Buenos Aires: Amorrortu, 1989.

HELLER, A.; FEHÉR, F. Biopolítica. La modernidad y la liberación del cuerpo, Barcelona: Península, 1995.

. Estigma, la identidad deteriorada. Buenos Aires: Amorrortu, 1996.

HALL, E. El lenguaje silencioso. Madrid: Alianza, 198I. . La dimensión oculta. Buenos Aires: Siglo XXI, 1987.

KNAPP, M. La comunicación no verbal. Barcelona: Paidós, 1992.

LE BOULCH, J. Hacia una ciencia del movimiento humano. Introducción a la psicokinética. Buenos Aires: Paidós, 1991. 
LE BRETÓN, D. Antropología del cuerpo y la modernidad. Buenos Aires: Nueva Visión, 1990. . Las pasiones ordinarias. Antropología de las emociones. Buenos Aires: Nueva Visión, 1999.

LIPOVETSKY, G. La era del vacío. Ensayos sobre individualismo contemporáneo. México, D.F: Anagrama, 1988.

MAFFESOLI, M. El instante eterno. El retorno de lo trágico en las sociedades posmodernas. Buenos Aires: Paidós, 2001.

. El nomadismo. Vagabundeos iniciáticos. D.F: Fondo de Cultura Económica, 2004.

MAINETTI, J. A. El tiempo biológico y el hombre. In: ROVALETTI, M. L. (Ed.). Temporalidad. El problema del tiempo en el pensamiento actual. Buenos Aires: Le Lugar Editorial, 1999.

MARGULIS, M. et al. Juventud, cultura, sexualidad. Buenos Aires: Biblos, 2003.

MARTÍN BARBERO, J. Jóvenes: des-orden cultural y palimpsestos de identidad. Revista Oficios Terrestres, La Plata, año IV, n. 5, pp. 64-72, ago. 1998.

OBIOLS, G. et alotros. Adolescencia, posmodernidad y escuela secundaria. Buenos Aires: Kapelusz, 1993.

ORTIZ, R. Mundialización y cultura. Buenos Aires: Editorial Alianza, 1997.

ORTIZ, R. Otro territorio. Bogotá: Convenio Andrés Bello, 1998.

PARLEBAS, P. Educación física moderna y ciencia de la acción motriz. In: I ER. Congreso de Educación Física y Ciencias, 1993, La Plata. Actas... La Plata: Departamento de Educación Física, FHCE, UNLP, I993. v. I, p. |29-| 45.

RAUCH, A. El cuerpo en la educación física. Buenos Aires: Kapelusz, 1985.

REGUILLO CRUZ, R. Emergencia de culturas juveniles. Estrategias del desencanto. Buenos Aires: Norma, 2000.

ROVALETTI, M. L. (Ed.). Corporalidad. La problemática del cuerpo en el pensamiento actual. Buenos Aires: Lugar Editorial, 1998.

(Ed.). Temporalidad. El problema del tiempo en el pensamiento actual. Buenos Aires: Le Lugar Editorial, 1999.

SARLO, B. Instantáneas. Medios, ciudad y costumbres en el fin de siglo. Buenos Aires: Ariel, 1996. 
SCHÜTZ, A.; LUCKMANN, T. Las estructuras del mundo de la vida. Buenos Aires: Amorrortu Editores, 1973.

SCHÜTZ, A. El problema de la realidad social. Buenos Aires: Amorrortu, 1974.

SCHVARSTEIN, L. Psicología social de las organizaciones. Nuevos aportes. Buenos Aires: Paidós, 1992. . Identidad de las organizaciones. Invarianza y cambio. Buenos Aires: Paidós, 1994

VÁZQUEZ GÓMEZ, B. La educación física en la educación básica. Madrid: Gymnos, 1989.

WOLF, M. Sociologías de la vida cotidiana. Madrid: Cátedra Teorema, 1979.

Recebido: 21 jul. 2009 Aprovado: I dez. 2009

Endereço para correspondência

Gabriel Armando Cachorro 68 N. 2205 e// 39 y I 40 La Plata CP 1900

Buenos Aires - Argentina 